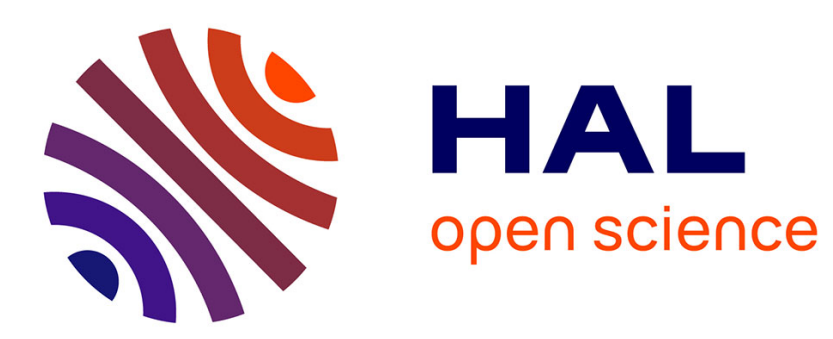

\title{
Mesure de l'endommagement anisotrope d'un composite céramique-céramique par une méthode ultrasonore
}

\author{
Stéphane Baste, Rachid El Guerjouma, Alain Gérard
}

\section{To cite this version:}

Stéphane Baste, Rachid El Guerjouma, Alain Gérard. Mesure de l'endommagement anisotrope d'un composite céramique-céramique par une méthode ultrasonore. Revue de Physique Appliquée, 1989, 24 (7), pp.721-731. 10.1051/rphysap:01989002407072100 . jpa-00246096

\section{HAL Id: jpa-00246096 https://hal.science/jpa-00246096}

Submitted on 1 Jan 1989

HAL is a multi-disciplinary open access archive for the deposit and dissemination of scientific research documents, whether they are published or not. The documents may come from teaching and research institutions in France or abroad, or from public or private research centers.
L'archive ouverte pluridisciplinaire HAL, est destinée au dépôt et à la diffusion de documents scientifiques de niveau recherche, publiés ou non, émanant des établissements d'enseignement et de recherche français ou étrangers, des laboratoires publics ou privés. 


\title{
Mesure de l'endommagement anisotrope d'un composite céramique- céramique par une méthode ultrasonore
}

\author{
Stéphane Baste, Rachid El Guerjouma et Alain Gérard \\ Laboratoire de Mécanique Physique, U.R.A. C.N.R.S. 867, 351 Cours de la Libération, 33405 Talence \\ Cedex, France
}

(Reçu le 23 décembre 1988, révisé et accepté le 21 mars 1989)

\begin{abstract}
Résumé. - L'endommagement en traction uniaxiale d'un composite céramique-céramique est analysé par une méthode interférométrique ultrasonore. La loi de comportement couplant endommagement anisotrope et élasticité anisotrope est obtenue en terme de contrainte par une équivalence en énergie dans le cadre de la thermodynamique des processus irréversibles. Dans le plan exploré contenant l'axe de la charge et la normale à l'éprouvette, la mesure des temps de vol, associée à une procédure d'optimisation, fournit, pour divers niveaux de contrainte, les variations des constantes élastiques, inaccessibles par des mesures classiques de déformations. Ces variations sont celles nécessaires à l'identification de l'endommagement anisotrope de ce plan principal (4 raideurs, 3 variables internes d'endommagement et leur loi d'évolution) et à la validation du modèle de comportement retenu.
\end{abstract}

\begin{abstract}
The damage of a Sic-Sic composite is evaluated during uniaxial tensile test using an ultrasonic interferometric method. The behaviour law coupling anisotropic elasticity and anisotropic damage is obtained in terms of stress by energy equivalence within the thermodynamics of irreversible processes. For the investigated plane containing the loading axis and the normal to the sample, a transit-time measurement method joined to an optimization process, provides, for various level of stress, the elastic constant variations, inaccessible by classical strain measurements. These variations are those necessary required to identify the anisotropic damage of this principal plane (4 stiffnesses, 3 damage parameters and their corresponding constitutive laws) and to validate the behaviour model.
\end{abstract}

\section{Introduction.}

Les composites à matrice céramique (C.M.C.) présentent souvent un comportement mécanique fortement non linéaire. Contrairement aux céramiques monolithiques pour lesquelles le comportement linéaire fragile traduit une propagation directe des fissures, les C.M.C. montrent des cheminements complexes de fissures, liés à la présence des fibres, leur conférant une ténacité et une résistance à la propagation de fissures élevées [1].

Les C.M.C. se classent dans les composites du type fragile-fragile pour lesquels l'allongement à rupture de la matrice est inférieur à celui des fibres. C'est le cas inverse de celui des composites à matrice polymère où la rupture est généralement gouvernée par les fibres. La rupture de la matrice précède celle des fibres. Cette «fracturation multiple » [2] de la matrice induit cette non-linéarité typique.
Cette microfissuration matricielle peut engendrer des déchaussements fibre-matrice et des glissements de la fibre dans la matrice. Ainsi, à partir de mêmes mécanismes élémentaires (microfissuration de la matrice, frottement sur les fissures d'interfaces fibrematrice ainsi créées), et suivant l'importance des effets de frottement, plusieurs types de comportement très différents peuvent être observés [3]. Ainsi la perte de rigidité, qui caractérise la microfissuration de la matrice, peut s'accompagner de déformations irréversibles et de boucles d'hystérésis [3-5].

Dès lors l'endommagement peut être évalué en terme de déformations résiduelles et d'énergie absorbée [5] ou bien en terme de variation du module d'Young suivant la direction du chargement [6], quantités mesurables par les techniques mécaniques conventionnelles telles que, par exemple, des extensomètres à pinces. Il convient de souligner que ces moyens de mesure n'autorisent que des évaluations 
du phénomène parallèlement à l'axe de la charge et donc ne permettent pas de mesure des modules d'Young dans les plans orthogonaux à l'axe de chargement ou de mesure des modules de cisaillement nécessaires à l'identification d'un endommagement anisotrope. Dans le cas des matériaux anisotropes, ceci oblige à effectuer des relevés sur un grand nombre d'éprouvettes (taillées suivant différents axes du composite) pour accéder à un maximum d'éléments du tenseur d'endommagement associé au comportement du matériau. A l'évidence, le grand nombre de manipulations nécessaires, sur des échantillons parfois difficilement comparables, induit une précision médiocre souvent liée à une dispersion importante des résultats.

Pour pallier à ces inconvénients, mesure uniquement parallèlement à l'axe de la charge en particulier, et contourner certains écueils (dispersion et manque de reproductibilité des résultats par exemple) d'autres techniques d'évaluation de l'endommagement doivent être imaginées. La caractérisation des milieux anisotropes par des méthodes ultrasonores $[7,8]$, est une voie possible pour la détermination de paramètres indispensables à l'identification précise de lois de comportement complexes (endommagement anisotrope couplé à l'élasticité anisotrope) [9]. Les résultats préliminaires, permettant la mesure d'un endommagement anisotrope d'un C.M.C., obtenus par cette méthode ultrasonore, sont présentés dans ce travail.

$\mathrm{Si}$, comme nous l'avons déjà signalé, la modélisation du comportement du matériau en terme de déformations est d'un usage commode pour les mesures «mécaniques » conventionnelles, il n'en va pas de même pour celles fondées sur la propagation ultrasonore [9]. Aussi, à partir de la thermodynamique des processus irréversibles, nous établissons (paragraphe 2) une formulation du comportement en termes de contraintes couplant l'élasticité anisotrope à l'endommagement anisotrope (formulation duale). Au paragraphe 3 nous présentons le matériau testé, le montage expérimental réalisé et quelques résultats préliminaires. Enfin nous précisons (paragraphe 4) la procédure employée pour déterminer les constantes d'élasticité du composite et leur variation au cours du chargement, qui nous donnent (paragraphe 5) l'évolution des composantes du tenseur d'endommagement retenu.

\section{Couplage endommagement anisotrope-élasticité anisotrope.}

Dans le cas unidimensionnel, il est désormais classique d'introduire un paramètre scalaire d'endommagement $d$ qui opère sur la contrainte appliquée $\sigma$ pour définir une contrainte effective [10] :

$$
\sigma_{\text {eff }}=\sigma(1-d)^{-1},
$$

qu'il faut appliquer à l'élément de volume vierge (non endommagé) pour obtenir la même déformation que celle provoquée par la contrainte $\sigma$ appliquée à l'élément de volume endommagé. Le paramètre $d$ a le sens physique d'une diminution de section résistante liée à la présence des microfissures. L'introduction de cette contrainte effective dans la loi constitutive de l'élasticité fournit une mesure indirecte de $d$ par des déchargements pendant l'essai d'écrouissage [11] :

$$
d=1-E \cdot\left(E^{0}\right)^{-1}
$$

où $E^{0}$ est le module d'Young initial et $E$ celui du matériau endommagé.

La généralisation tridimensionnelle conduit au tenseur des contraintes effectives $\boldsymbol{\sigma}_{\text {eff }}$ relié au tenseur des contraintes $\sigma$ par un opérateur $M(D)$ tel que :

$$
\boldsymbol{\sigma}_{\text {eff }}=M(\mathbf{D})[\boldsymbol{\sigma}] .
$$

La variable d'endommagement D doit avoir un. caractère tensoriel pour rendre compte de l'orientation privilégiée des microfissures. La loi de comportement s'obtient en substituant dans le potentiel élastique le tenseur des contraintes effectives au tenseur des contraintes appliquées, ce qui assure la symétrie de l'opérateur d'élasticité du matériau endommagé [12]. Cette équivalence en énergie de déformation fournit la relation entre les tenseurs des souplesses $\mathbf{S}$ et $\mathbf{S}_{0}$ du matériau endommagé et du matériau vierge par:

$$
\mathbf{S}(\mathrm{D})=M^{\mathrm{t}}(\mathrm{D}): \mathbf{S}_{0}: M(\mathrm{D}),
$$

où l'exposant $\mathrm{t}$ désigne la transposition habituelle et : le produit tensoriel.

On obtient ainsi l'expression de la loi d'élasticité du matériau endommagé reliant $\boldsymbol{\sigma}$ au tenseur des déformations élastiques $\varepsilon^{\mathrm{e}}$. Comme nous l'avons déjà indiqué, une telle expression est mal adaptée aux techniques ultrasonores que nous allons utiliser. Une formulation duale doit être recherchée. L'une des possibilités consiste à partir de l'enthalpie libre et à étendre à ce cadre la démarche précédente. En introduisant le tenseur des déformations élastiques effectives $\varepsilon_{\text {eff }}^{\mathrm{e}}$, relié au tenseur des déformations élastiques par:

$$
\varepsilon_{\text {eff }}^{\mathrm{e}}=\left(M^{\mathrm{t}}(\mathbf{D})\right)^{-1}\left[\boldsymbol{\varepsilon}^{\mathrm{e}}\right],
$$

l'équivalence en énergie [6] exprimée en contrainte fournit alors la relation entre les tenseurs des raideurs $\mathbf{C}$ et $\mathbf{C}_{0}$ du matériau endommagé et du matériau vierge :

$$
\mathbf{C}(\mathbf{D})=(M(\mathbf{D}))^{-1}: \mathbf{C}_{0}:\left(M^{\mathrm{t}}(\mathbf{D})\right)^{-1} .
$$


On obtient, alors, la loi d'élasticité du matériau endommagé reliant $\varepsilon^{\mathrm{e}}$ et $\boldsymbol{\sigma}$ :

$\boldsymbol{\sigma}=\mathbf{C}(\mathrm{D})\left[\varepsilon^{\mathrm{e}}\right]=(M(\mathrm{D}))^{-1}: \mathbf{C}_{0}:\left(M^{\mathrm{t}}(\mathrm{D})\right)^{-1}\left[\varepsilon^{\mathrm{e}}\right]$.

Nous choisissons pour D, un tenseur diagonal d'ordre quatre [6] qui privilégie les axes de symétrie du composite. Notons que les théories de l'homogénéisation montrent qu'une microfissuration périodique, comportant des plans de symétrie, peut être décrite par un tenseur d'endommagement d'ordre quatre [13]. Parmi plusieurs possibilités [14], nous choisissons l'opérateur $M(D)$ tel que :

$$
\varepsilon_{\mathrm{eff}}^{e}=(1-D)^{1 / 2}: \varepsilon^{e}:(1-D)^{1 / 2},
$$

qui amène à un tenseur des déformations élastiques effectives symétrique. La loi d'élasticité (7) est orthotrope par rapport aux directions principales de symétrie du composite endommagé. Les éléments $C_{i j}$ non nuls de la matrice d'élasticité s'écrivent :

$$
\begin{aligned}
& C_{11}=C_{11}^{0}\left(1-D_{1}\right)^{2} ; \quad C_{44}=C_{44}^{0}\left(1-D_{4}\right)^{2} ; \\
& C_{22}=C_{22}^{0}\left(1-D_{2}\right)^{2} ; \quad C_{55}=C_{55}^{0}\left(1-D_{5}\right)^{2} ; \\
& C_{33}=C_{33}^{0}\left(1-D_{3}\right)^{2} ; \quad C_{66}=C_{66}^{0}\left(1-D_{6}\right)^{2} ; \\
& C_{12}=C_{21}=C_{12}^{0}\left(1-D_{1}\right)\left(1-D_{2}\right) ; \\
& C_{13}=C_{31}=C_{13}^{0}\left(1-D_{1}\right)\left(1-D_{3}\right) ; \\
& C_{23}=C_{32}=C_{23}^{0}\left(1-D_{3}\right)\left(1-D_{2}\right) ;
\end{aligned}
$$

où $C_{i j}^{0}$ sont les composantes de la matrice des raideurs du matériau vierge et $D_{i}$ celles du tenseur d'endommagement. Ces relations entre caractéristiques élastiques du matériau vierge (notées par l'indice supérieur 0) et celles du matériau endommagé permettent, pour un chargement quelconque, la détermination complète des éléments $D_{i}(i=1$ à 6) du tenseur d'endommagement, soit :

$$
\begin{aligned}
& D_{1}=1-\left(\frac{C_{11}}{C_{11}^{0}}\right)^{1 / 2}, \quad D_{4}=1-\left(\frac{C_{44}}{C_{44}^{0}}\right)^{1 / 2}, \\
& D_{2}=1-\left(\frac{C_{22}}{C_{22}^{0}}\right)^{1 / 2}, \quad D_{5}=1-\left(\frac{C_{55}}{C_{55}^{0}}\right)^{1 / 2}, \\
& D_{3}=1-\left(\frac{C_{33}}{C_{33}^{0}}\right)^{1 / 2}, \quad D_{6}=1-\left(\frac{C_{66}}{C_{66}^{0}}\right)^{1 / 2} .
\end{aligned}
$$

Il est à noter que, contrairement aux méthodes conventionnelles de mesure de déformations pour lesquelles les mesures indirectes de l'endommagement reposent sur les variations des coefficients de Poisson [6], les variations des éléments non diagonaux de la matrice des rigidités ne sont plus indispensables à l'évaluation du dommage mais doivent seulement être vérifiées pour s'assurer de la validité du modèle.
Enfin d'après (9) et les relations entre constantes de l'ingénieur du matériau vierge (notées par l'indice supérieur 0) et celles du matériau endommagé [6] :

$$
\begin{aligned}
& E_{1}=E_{1}^{0}\left(1-D_{1}\right)^{2} ; \quad G_{23}=G_{23}^{0}\left(1-D_{4}\right)^{2} ; \\
& E_{2}=E_{2}^{0}\left(1-D_{2}\right)^{2} ; \quad G_{13}=G_{13}^{0}\left(1-D_{5}\right)^{2} ; \\
& E_{3}=E_{3}^{0}\left(1-D_{3}\right)^{2} ; \quad G_{12}=G_{12}^{0}\left(1-D_{6}\right)^{2} ; \\
& \nu_{12}=\nu_{12}^{0}\left(1-D_{1}\right)\left(1-D_{2}\right)^{-1} ; \\
& \nu_{13}=\nu_{13}^{0}\left(1-D_{1}\right)\left(1-D_{3}\right)^{-1} ; \\
& \nu_{23}=\nu_{23}^{0}\left(1-D_{2}\right)\left(1-D_{3}\right)^{-1} ;
\end{aligned}
$$

on peut remarquer que la mesure de la variation des $C_{i i}$ donne, de fait, la mesure de la variation des $E_{i}$ et $\operatorname{des} G_{i j}$.

\section{Résultats expérimentaux.}

Le composite Sic-Sic est fabriqué par empilement de tissus bidirectionnels de carbure de silicium, densifiés en phase gazeuse par la matrice de carbure de silicium. Le matériau a été testé en traction suivant la direction 1 . Les directions équivalentes 1 et 2 orthogonales sont dans le ṕlan des tissus et correspondent aux directions chaînes et trames. La direction 3 est orthogonale au plan des tissus (Fig. 1).

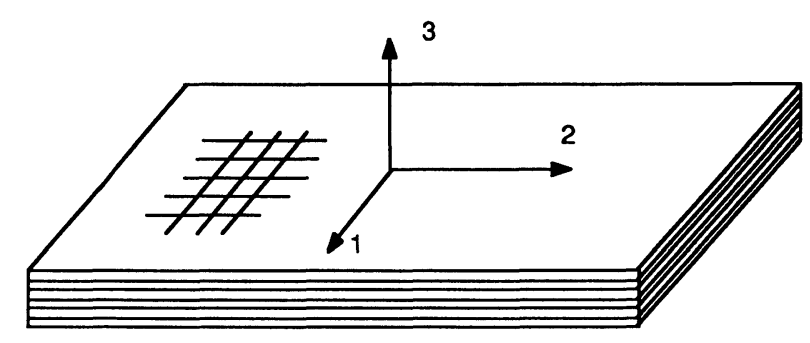

Fig. 1. - Axes principaux du composite.

[Composite principal axis.]

L'échantillon a été précontraint au moyen d'une machine de traction jusqu'à $75 \mathrm{MPa}$, limite du domaine pseudo-linéaire (Fig. 2). Aucune évolution significative des propriétés élastiques n'a été décelée par une mesure du module d'Young par extensomètres à pinces et, après déchargement total et démontage de l'éprouvette, par une mesure au spectrointerféromètre à immersion [8]. Cela confirme, comme pour les moyens de mesures mécaniques conventionnels, la nécessité d'une évaluation sous charge de l'endommagement en particulier lorsque les microfissures se referment lors d'un déchargement. Bien que l'échantillon ait subi un chargementdéchargement à la limite du domaine élastique, et 


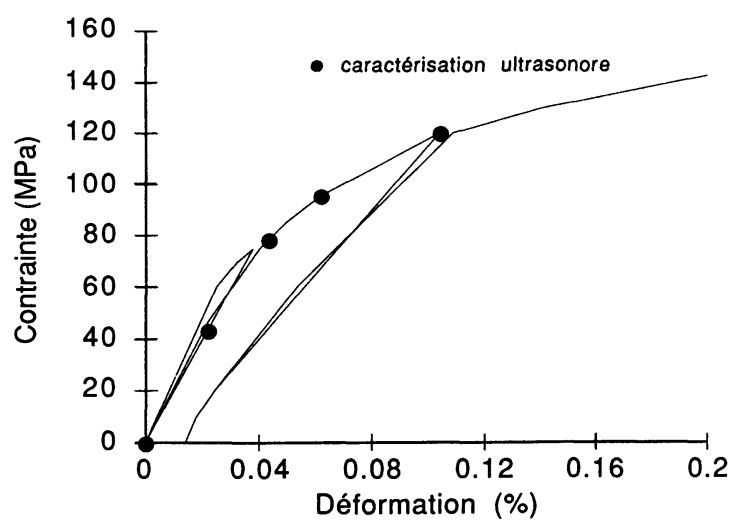

Fig. 2. - Courbe contrainte-déformation en traction suivant l'axe 1 .

[Tensile stress-strain curve in the 1 direction.]

donc a été probablement déjà endommagé, cet état du matériau sera considéré dans la suite comme l'état de référence.

A chaque niveau de contrainte, la caractérisation de l'endommagement est fondée sur la mesure de la vitesse des ondes de volume transitant dans l'éprouvette. Pour accéder aux vitesses de propagation des ondes au sein du matériau en fonction de l'angle d'incidence (ou de transmission) et de la contrainte, une installation complexe a été mise au point au laboratoire. La figure 3 en présente le synoptique. Cette installation est composée d'un banc de caractérisation ultrasonore associé à une machine de traction de faible volume. La machine de traction proprement dite est un piston actionné par une pompe manuelle, développant une force de 0 à

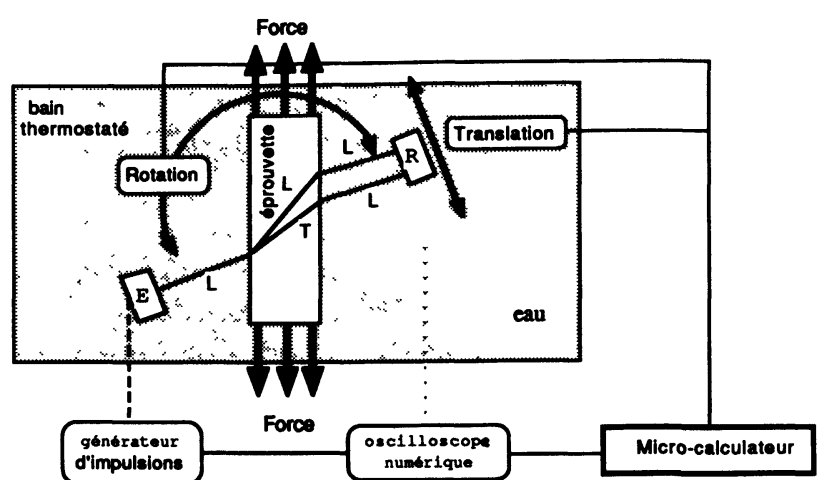

Fig. 3. - Banc de caractérisation ultrasonore sous charge [Under load ultrasonic characterization device.]

15 tonnes sur une éprouvette solidaire du socle de la machine et fixée sur la tige du piston. Le banc de caractérisation ultrasonore est similaire à celui élaboré par Hosten et al. [7]. Cette dernière version est modifiée et adaptée à la caractérisation de matériaux sous charge. La méthode utilisée pour la détermination des vitesses est fondée sur la mesure du temps de vol par corrélation-filtrage, la mesure de l'atténuation s'obtient en faisant le rapport des densités spectrales de puissance du signal de mesure et du signal de référence acquis en l'absence de l'échantillon [8].

Les résultats expérimentaux, portant sur un composite Sic-Sic, sont consignés dans les figures 4 et 5 . La fréquence centrale des transducteurs est de 2,25 MHz. La figure 4 (resp. 5) présente l'évolution des vitesses (resp. atténuations) des ondes longitudi-

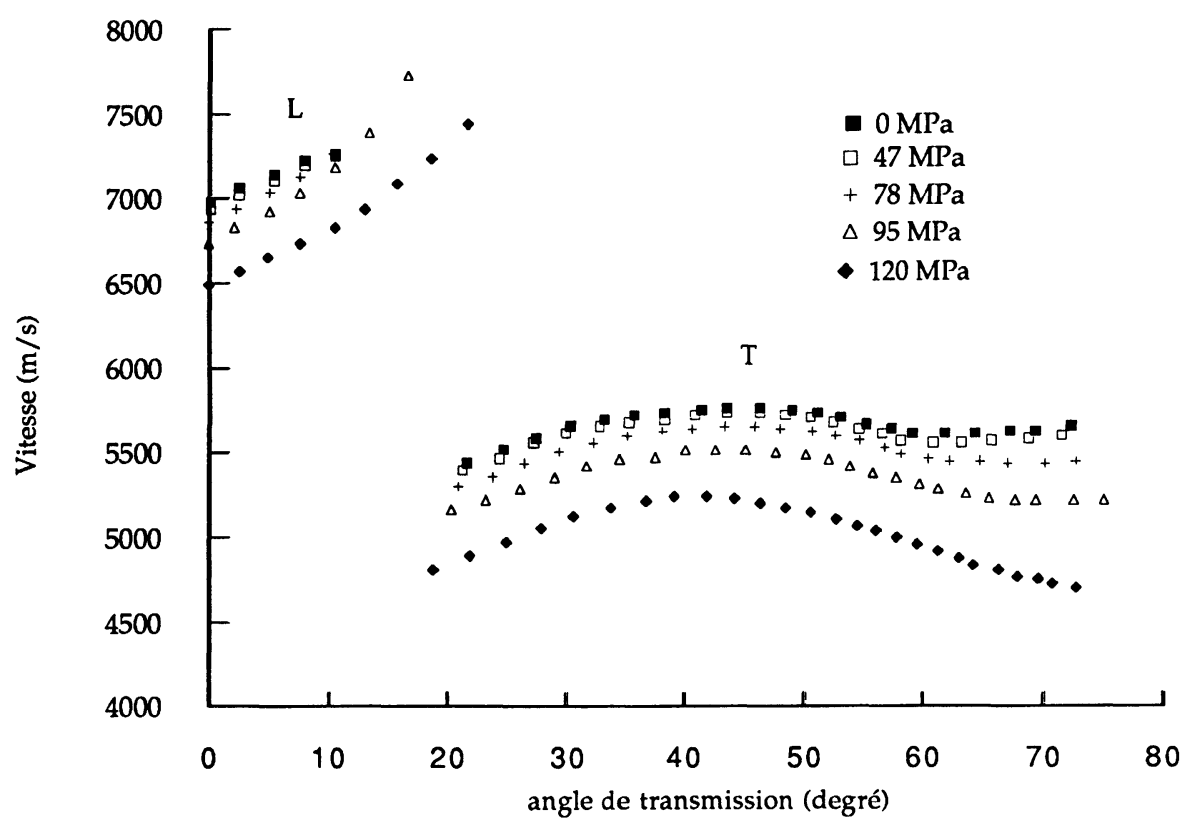

Fig. 4. - Vitesses de propagation en fonction de l'angle de transmission et de la contrainte.

[Propagation velocities versus transmission angle and stress.] 
nales (notées $\mathrm{L}$ ) et transversales (notées $\mathrm{T}$ ) en fonction de l'angle de transmission (resp. incident) et pour 5 niveaux de contrainte différents notés (•) sur la figure 2.

L'évolution des vitesses de propagation est importante. Elles diminuent continûment avec la charge. Ces variations permettront dans les paragraphes suivants l'évaluation de l'endommagement par le biais d'une procédure d'optimisation des constantes élastiques à partir des mesures des vitesses de propagation des ondes élastiques.

L'atténuation paraît être un indicateur plus sensible au dommage que les vitesses. En effet, la variation des coefficients de transmission déterminée expérimentalement (Fig. 5) est liée à l'évolution de l'atténuation due à l'endommagement et aux pertes interfaciales. La connaissance des constantes élastiques autorise le calcul des pertes aux interfaces et permet donc d'isoler ces dernières et d'accéder à l'atténuation [15]. Cependant, son lien avec la loi de comportement du milieu testé est loin d'être aussi évident que celui des variations de vitesse. Ainsi, les mesures d'atténuation ne sont-elles, pour l'instant, qu'au stade d'une évaluation qualitative de l'endommagement. Les coefficients de transmission, qui décroissent en fonction de la contrainte appliquée, traduisent classiquement une augmentation de la densité des micro-défauts. On retrouve ici le sens physique de la variable interne d'endommagement (diminution de la section résistante liée à la présence de micro-fissures). On note sur la figure 5 une évolution importante, au cours du chargement, de la courbe d'atténuation des ondes transversales en fonction de l'angle de propagation, attestant d'une modification de la structure interne (géométrie des micro-défauts) du matériau. Sur cette figure apparaissent nettement les angles limites relatifs aux modes quasi-longitudinaux puis quasi-transversaux au-delà desquels ces ondes ne sont plus engendrées dans l'échantillon.

\section{Détermination des constantes d'élasticité.}

L'exploitation des résultats expérimentaux, dont quelques exemples ont été donnés au paragraphe précédent, passe nécessairement par la connaissance des relations reliant la vitesse des ondes ultrasonores aux caractéristiques mécaniques intervenant dans la loi de comportement du milieu support de la propagation. Celles-ci s'obtiennent en introduisant l'expression du champ des déplacements associé à une onde plane monochromatique dans les équations du mouvement d'un milieu continu. Le matériau étant, a priori, réputé homogène à la fréquence utilisée, les modes propagés sont les solutions de l'équation de propagation [16]. A la masse volumique près, les valeurs propres de l'équation caractéristique associée sont les carrés des vitesses de propagation $\left(V_{\mathrm{L}}\right.$ ou $\left.V_{\mathrm{T}}\right)$, ce qui, pour un matériau orthotrope ( 9 constantes d'élasticité indépendantes), dans le plan $(3,1)$ considéré ici, conduit à :

$$
2 \rho \cdot V_{\mathrm{L}, \mathrm{T}}^{2}=A \pm\left(A^{2}-4 B\right)^{1 / 2},
$$

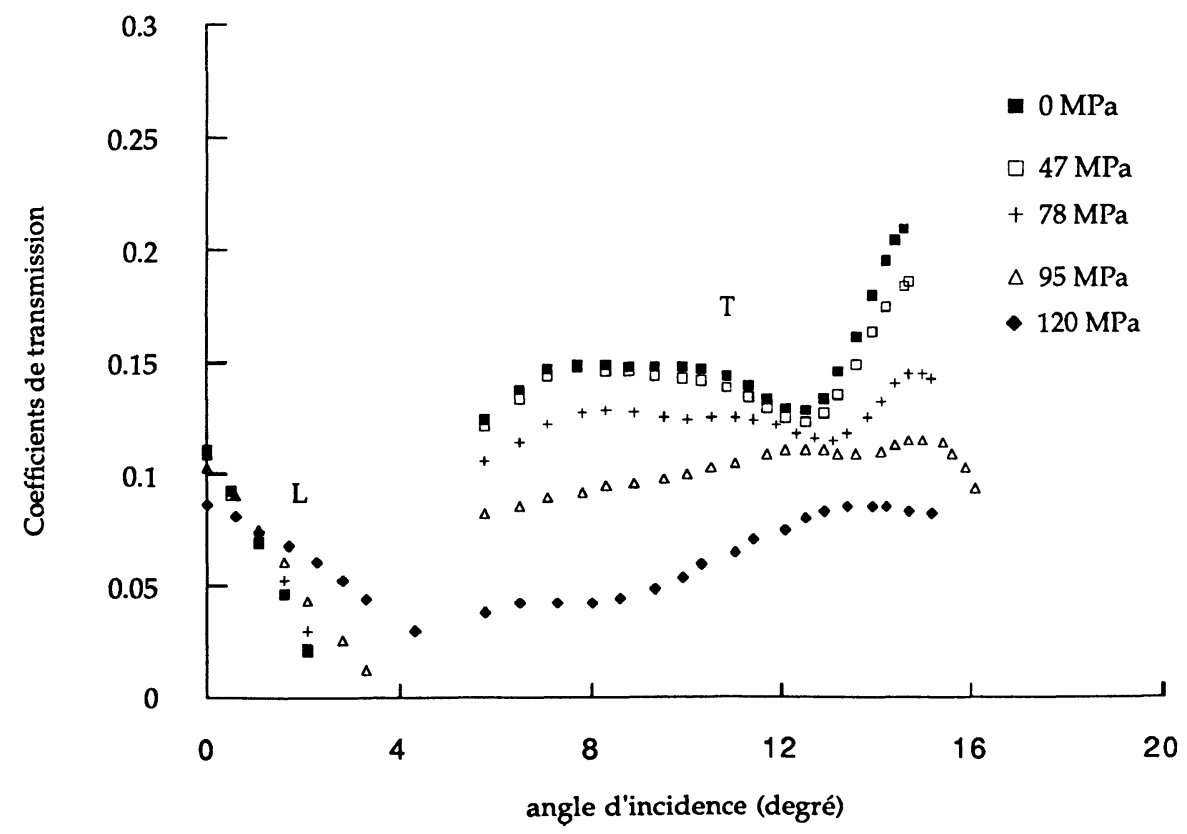

Fig. 5. - Coefficients de transmission en fonction de l'angle d'incidence et de la contrainte.

[Transmission coefficients versus incidence angle and stress.] 
avec

$A=C_{33} \cos ^{2} \theta+C_{11} \sin ^{2} \theta+C_{55}$,

$B=\left(C_{33} \cos ^{2} \theta+C_{55} \sin ^{2} \theta\right)\left(C_{55} \cos ^{2} \theta+\right.$

$$
\left.+C_{11} \sin ^{2} \theta\right)-\left(C_{13}+C_{55}\right)^{2} \cos ^{2} \theta \sin ^{2} \theta,
$$

$\boldsymbol{\theta}$ désignant l'angle de transmission dans le matériau et l'indice $\mathrm{L}$ (resp. $\mathrm{T}$ ) précisant le mode de propagation quasi-longitudinal (resp. quasi-transversal).

Une procédure d'optimisation [17] de toutes les valeurs expérimentales de vitesse, utilisant la méthode de Newton, permet d'en déduire les valeurs des raideurs du matériau à chaque niveau de chargement. Celles-ci sont consignées sur les figures $6 a$ à

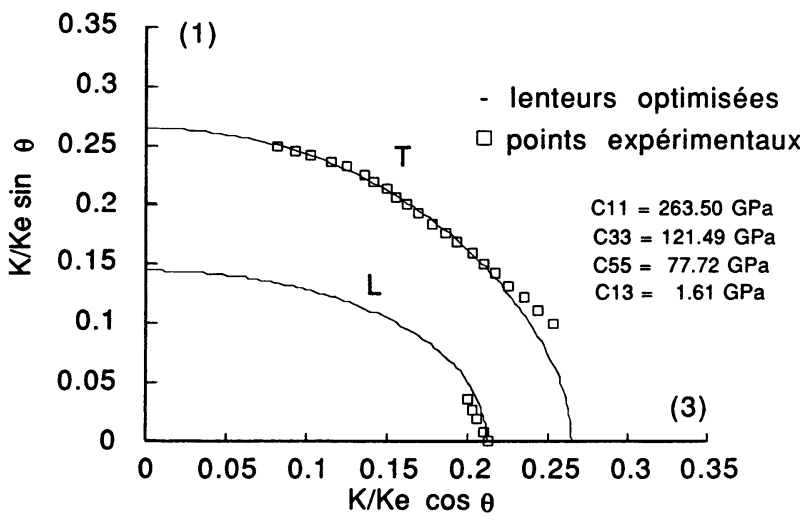

a)

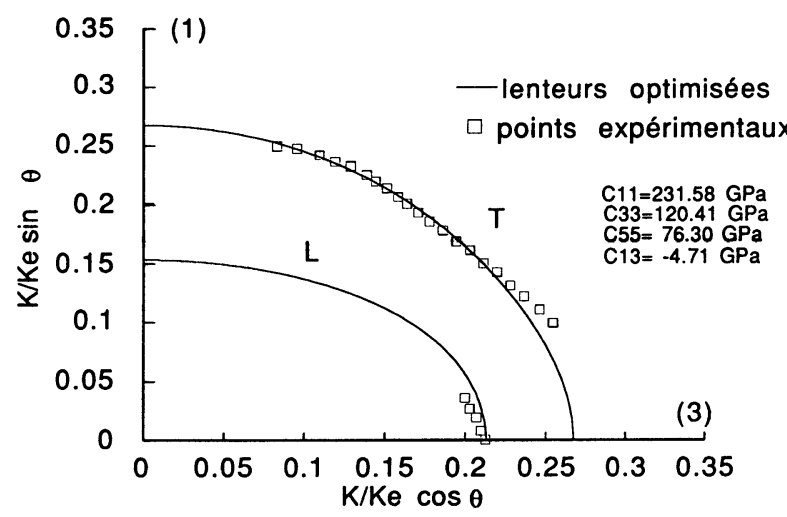

b)

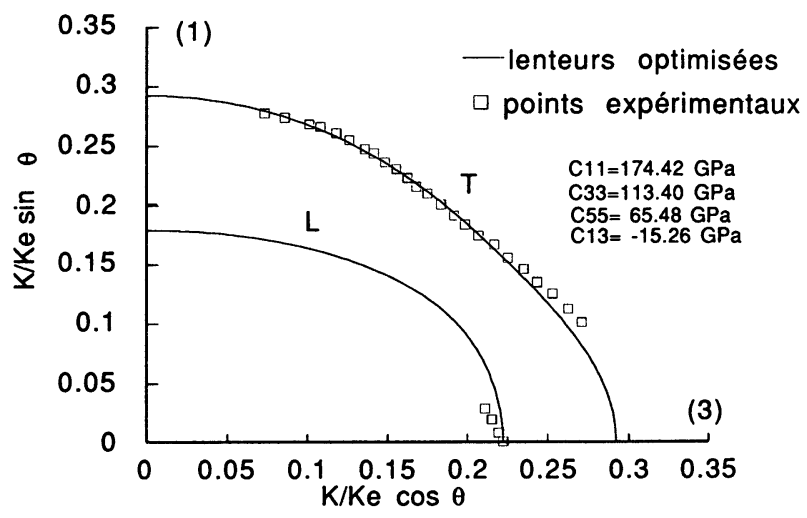

d)

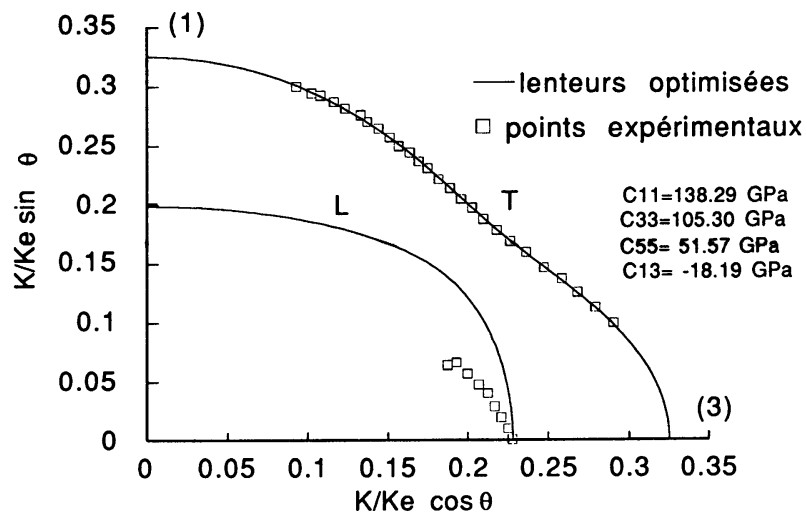

e)

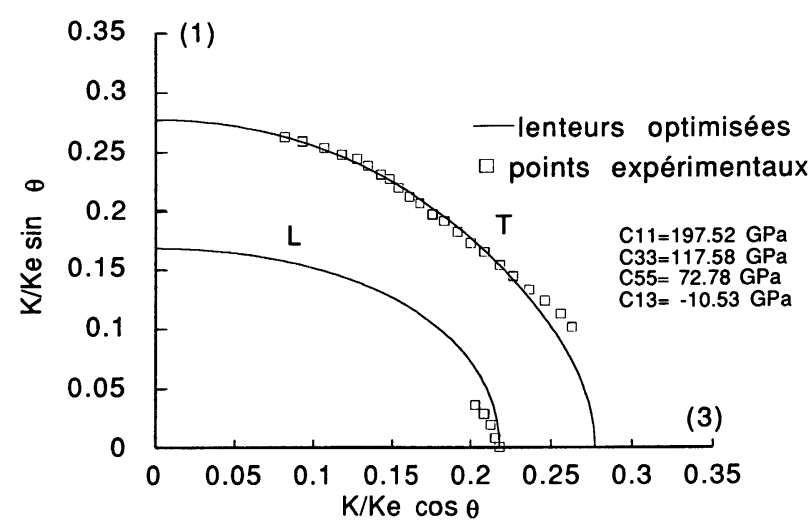

c)

Fig. 6. - Constantes élastiques et courbes de lenteurs normalisées du composite - plan $(1,3)$ anisotrope - (a) à contrainte nulle $0 \mathrm{MPa}$, (b) pour une contrainte de $47 \mathrm{MPa}$, (c) pour une contrainte de $78 \mathrm{MPa}$, (d) pour une contrainte de $95 \mathrm{MPa}$, (e) pour une contrainte de $120 \mathrm{MPa}$.

[Stiffness coefficients and normalized slowness curves in the anisotropic $(1,3)$ plane : (a) for zero stress, (b) for $47 \mathrm{MPa}$, (c) for $78 \mathrm{MPa}$, (d) for $95 \mathrm{MPa}$, (e) for $120 \mathrm{MPa}$.] 
6e. Les courbes apparaissant sur ces figures correspondent aux courbes des lenteurs (l'inverse des vitesses) associées à (12) ou plus précisément au tracé de l'évolution des nombres d'ondes $\left(k_{\mathrm{L}}\right.$ onde quasi-longitudinale, $k_{\mathrm{T}}$ onde quasi-transversale) dans le matériau normalisés par le nombre d'onde dans l'eau $k_{\mathrm{e}}$ en fonction de l'angle de réfraction $\theta$. La vitesse du son dans l'eau étant de $1497 \mathrm{~m} / \mathrm{s}$ au moment des relevés expérimentaux. Dans chacune des figures $6 \mathrm{a}$ à $6 \mathrm{e}$ les $(\square)$ correspondent aux points expérimentaux et les lignes continues au tracé des courbes de lenteur exécuté à l'aide des valeurs des $C_{i j}$ optimisées. Ainsi est visualisé l'écart quadratique entre points expérimentaux et points calculés. Les divergences que l'on peut constater entre points expérimentaux et courbes optimisées sont en majorité attribuables aux mélanges des modes quasilongitudinaux et quasi-transversaux dans la fenêtre de mesure [18]. En fonction du chargement, la séparation de ces modes est variable et les erreurs se reportent préférentiellement soit sur le mode quasitransversal (Fig. 6a) soit sur le mode quasi-longitudinal (Fig. 6e) soit sur les deux modes (Fig. 6c).

L'évolution de l'anisotropie du composite en fonction de la charge qui lui est appliquée est donnée par le faisceau des courbes de lenteurs regroupées à la figure 7. On note que les courbes de lenteurs ne subissent pas une simple homothétie mais se déforment lorsque la contrainte augmente. Ceci traduit l'effet d'un endommagement anisotrope qui influence différemment chaque raideur et que nous identifions dans le paragraphe suivant.

\section{Evolution de l'endommagement.}

Les relevés expérimentaux effectués dans le plan $(1,3)$ donnent accès aux raideurs $C_{11}, C_{33}, C_{55}$ et
$C_{13}$ évaluées au paragraphe précédent à chaque niveau de charge. Les courbes représentatives de leur évolution en fonction du chargement sont données figure 8. Comme on s'y attendait, on

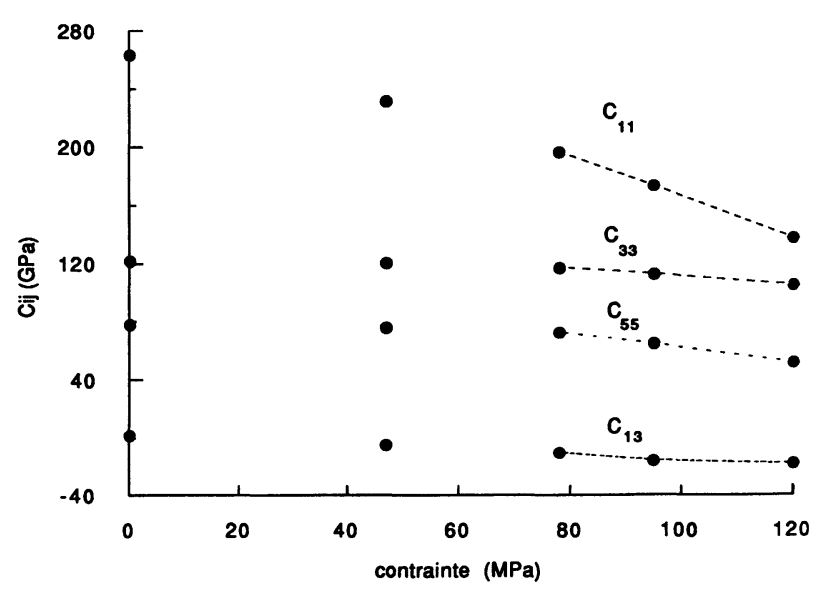

Fig. 8. - Variation des constantes d'élasticité.

[Stiffness coefficients variation.]

constate une perte de rigidité importante de l'échantillon suivant l'axe de traction de l'ordre de $120 \mathrm{GPa}$ et une variation plus faible, environ $20 \mathrm{GPa}$, pour les autres éléments du tenseur des rigidités accessibles aux mesures. On observe que le $C_{13}$ devient négatif. Les variations de cette constante non diagonale, apparemment inattendues, mais qui sont admissibles puisque le mineur,

$$
M_{13}=C_{11} C_{33}-C_{13}^{2},
$$

reste toujours positif (l'opérateur d'élasticité doit demeurer quadratique défini positif), sont tout-à-fait

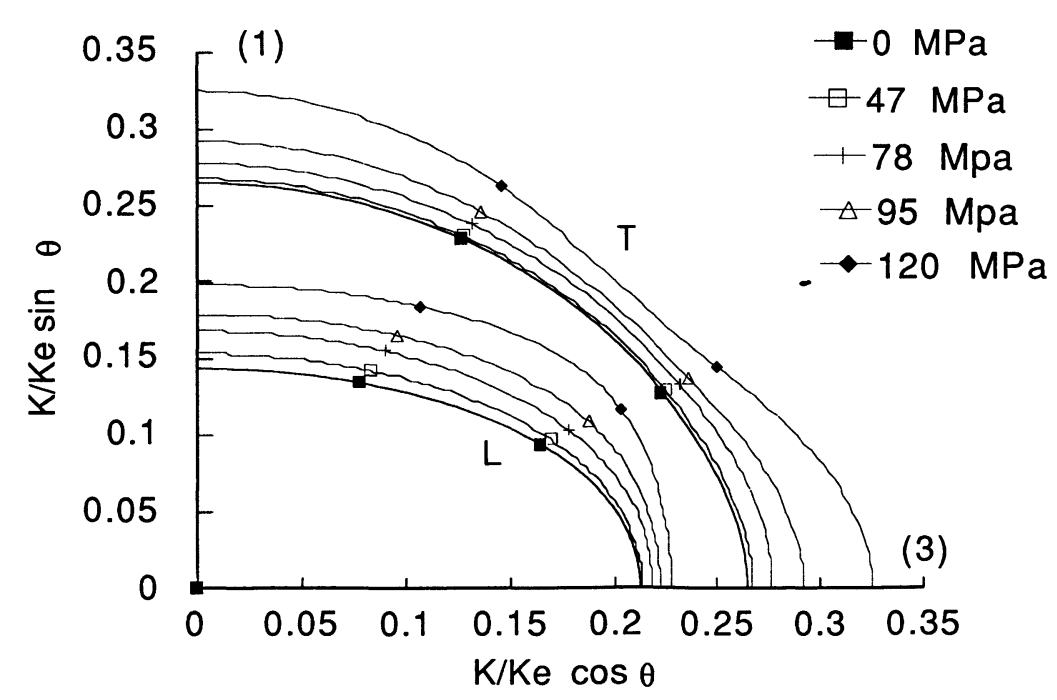

Fig. 7. - Evolution des courbes de lenteurs normalisées du composite en fonction de la contrainte, plan $(1,3)$ anisotrope.

[Composite slowness curves evolution as function of stress, anisotropic $(1,3)$ plane.] 
significatives de phénomènes de délaminage dans le plan $(1,3)$ au sein de ce composite fragile-fragile 2D.

Dès lors, l'utilisation des relations (10) fournit trois composantes $D_{1}, D_{3}$ et $D_{5}$ du tenseur d'endommagement consignées dans la figure 9. Ces trois variables internes vont croissant avec le chargement et leur évolution différente est révélatrice d'un endommagement anisotrope. Pour la plage de

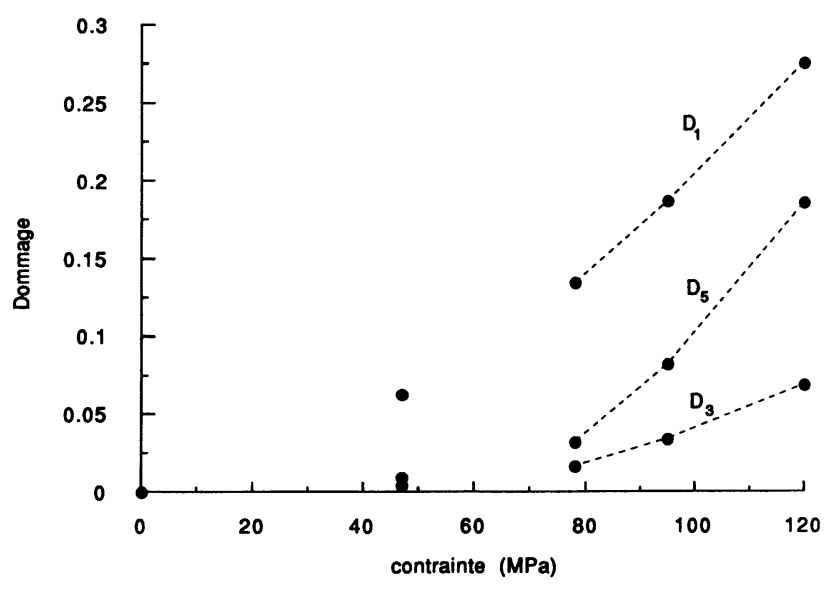

Fig. 9. - Evolution des composantes du tenseur d'endommagement.

[Damage tensor component evolution.]

mesure retenue, l'endommagement le plus (resp. moins) important a lieu logiquement selon l'axe $1 \mathrm{de}$ chargement (resp. 3). Par ailleurs la variable $D_{5}$ a une croissance plus rapide que les deux autres $\left(D_{1}\right.$ et $\left.D_{3}\right)$ ce qui est bien conforme au pressentiment d'un endommagement plus catastrophique en cisaillement dans le plan $(1,3)$ (ou $(2,3)$ ) que dans le plan translaminaire $(1,2)$. Ainsi, il est prévisible que la rupture de l'échantillon s'effectuera en cisaillement interlaminaire dans le plan $(1,3)$. Ceci dit, il faut se souvenir que nous avons considéré comme vierge un matériau dont le seuil d'endommagement a déjà certainement été franchi puisque chargé à $75 \mathrm{MPa}$, puis déchargé préalablement aux mesures ultrasonores. L'évolution mesurée des variables d'endommagement de la figure 9 n'est pas représentative de l'évolution associée à une montée en charge strictement monotone sur la courbe d'écrouissage [19], mais de celle associée à un chargement précédé du cycle charge-décharge à $75 \mathrm{MPa}$ (Fig. 2). Pour ce matériau, on observe généralement une fermeture des micro-fissures lors d'un déchargement. Les mesures ultrasonores à contrainte nulle concernent un matériau dont les fissures sont refermées : les constantes élastiques effectives sont identiques à celles du matériau vierge (à l'exception sans doute du $\left.C_{13}\right)$. A $47 \mathrm{MPa}$, les fissures préexistantes, créées par la précontrainte de $75 \mathrm{MPa}$, commencent à s'ouvrir de nouveau. Le niveau de précontrainte atteint, on revient sur la courbe d'écrouissage monotone. Par conséquent, si le niveau du seuil d'endommagement n'a pas été déterminé précisément, l'évolution du dommage est, après $75 \mathrm{MPa}$, conforme à celle d'une montée en charge monotone.

Cependant, comme nous l'avons déjà indiqué, la validité du modèle ne doit pas être oubliée. D'après les relations (9), la validité du modèle, pour le plan d'auscultation $(1,3)$ considéré, sera assurée si la variation de l'élément non diagonal $C_{13}$ est proportionnelle à celles des rigidités selon les axes 1 et 3 . En fait, le modèle retenu est expérimentalement vérifié si l'évolution du mineur $M_{13}$, qui doit rester positif, correspond à une décroissance telle que :

$$
\begin{aligned}
\delta M_{13} & \frac{C_{13}^{2}}{C_{11} C_{33}}=\frac{C_{13}^{02}\left(1-D_{1}\right)^{2}\left(1-D_{3}\right)^{2}}{C_{11}^{0} C_{33}^{0}\left(1-D_{1}\right)^{2}\left(1-D_{3}\right)^{2}} \\
= & \frac{C_{13}^{02}}{C_{11}^{0} C_{33}^{0}}=\mathrm{Cte} .
\end{aligned}
$$

La positivité du mineur $M_{13}$ est satisfaite mais pas la proportionnalité de $C_{13}^{2}$ et du produit $C_{11} C_{33}$. La figure 10 montre l'écart entre les valeurs expérimentales $\delta M_{13}$ extraites de nos mesures à divers niveaux de chargement et sa valeur initiale dont le modèle ne prévoit pas de variation. Il convient cependant d'observer que cet écart, en valeur absolue, reste faible (de l'ordre de $2 \%$ au plus). En revanche,

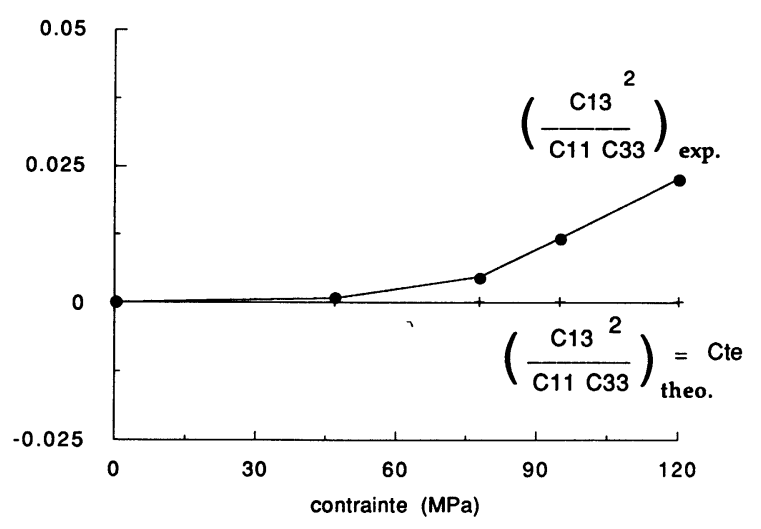

Fig. 10. - Ecart entre expérience et prévision du modèle.

[Deviation between experiment and model prevision.]

reporté sur l'évolution de la constante $C_{13}$, l'écart entre sa valeur expérimentale $C_{13}$ et sa valeur « théorique " $C_{13}^{0}\left(1-D_{1}\right)\left(1-D_{3}\right)$ est important (Fig. 11). On retrouve par cet écart le fait que l'état initial est en réalité un état déjà endommagé conformément à la précharge de $75 \mathrm{MPa}$ qui a modifié la valeur initiale $C_{13}^{0}$.

Pour tenter de décrire convenablement l'évolution expérimentale de $C_{13}$, il est donc nécessaire d'introduire une correction sur sa valeur initiale $C_{13}^{0}$. Pour 
ce faire, on "norme » les valeurs expérimentales $C_{13}$ par rapport au mineur $M_{13}$ de telle sorte que :

$$
\bar{C}_{13}=C_{13}+\left(C_{11}^{0} C_{33}^{0} C_{13}^{0-2}\right)^{1 / 2} \cdot C_{13}^{0-1},
$$

Dès lors, avec la figure 11 , on constate que l'évolution expérimentale de $\bar{C}_{13}$ satisfait quasiment la relation :

$$
\bar{C}_{13}=\bar{C}_{13}^{0}\left(1-D_{1}\right)\left(1-D_{3}\right),
$$

ce qui permet d'établir la relation donnant l'évolution de $C_{13}$ :

$$
\begin{aligned}
C_{13} & =\left[C_{13}^{0}+\frac{\left(C_{11}^{0} C_{33}^{0} C_{13}^{0-2}\right)^{1 / 2}}{C_{13}^{0}}\right] \times \\
& \times\left(1-D_{1}\right)\left(1-D_{3}\right)-\frac{\left(C_{11}^{0} C_{33}^{0} C_{13}^{0-2}\right)^{1 / 2}}{C_{13}^{0}}
\end{aligned}
$$

En posant :

$$
\begin{aligned}
C_{13 \text { corr. }}=C_{13}^{0}+ & \left.\frac{\left(C_{11}^{0} C_{33}^{0} C_{13}^{0-2}\right)^{1 / 2}}{C_{13}^{0}}\right) \times \\
& \times\left(\frac{D_{1} D_{3}-D_{1}-D_{3}}{\left(1-D_{1}\right)\left(1-D_{3}\right)}\right),
\end{aligned}
$$

la variation de la constante non diagonale, $C_{13}=C_{13 \text { corr. }}\left(1-D_{1}\right)\left(1-D_{3}\right)$, est alors en bon accord avec les relevés expérimentaux (Fig. 11).
Finalement, les relations entre les composantes de la matrice des raideurs du matériau vierge et celles du matériau endommagé accessibles par les mesures dans le plan d'investigation $(1,3)$ s'écrivent :

$$
\begin{aligned}
& C_{11}=C_{11}^{0}\left(1-D_{1}\right)^{2} ; \quad C_{33}=C_{33}^{0}\left(1-D_{3}\right)^{2} ; \\
& C_{55}=C_{55}^{0}\left(1-D_{5}\right)^{2}, \\
& C_{13}=\left[C_{13}^{0}+\left(\frac{\left(C_{11}^{0} C_{33}^{0} C_{13}^{0-2}\right)^{1 / 2}}{C_{13}^{0}}\right) \times\right. \\
& \left.\times\left(\frac{D_{1} D_{3}-D_{1}-D_{3}}{\left(1-D_{1}\right)\left(1-D_{3}\right)}\right)\right]\left(1-D_{1}\right)\left(1-D_{3}\right) .
\end{aligned}
$$

\section{Discussion et conclusion.}

La métrologie mécanique classique (jauge de déformation, extensomètre à pinces, ...) n'autorise que des mesures de déformation dans l'axe et orthogonalement à l'axe de la charge. Ce manque de souplesse est à l'origine du faible nombre de paramètres de raideur et d'endommagement évalués à partir d'une seule éprouvette. Pour tenter de cerner au mieux le phénomène de dommage tridimensionnel des matériaux, des modèles de plus en plus sophistiqués sont - élaborés pour reporter sur les variables d'endommagement le maximum d'informations accessibles dans les directions de mesures. C'est ainsi que sont apparus des modèles couplant endommagement anisotrope et élasticité isotrope ou non $[12,13,6]$ ou bien encore couplant endommagement anisotrope,

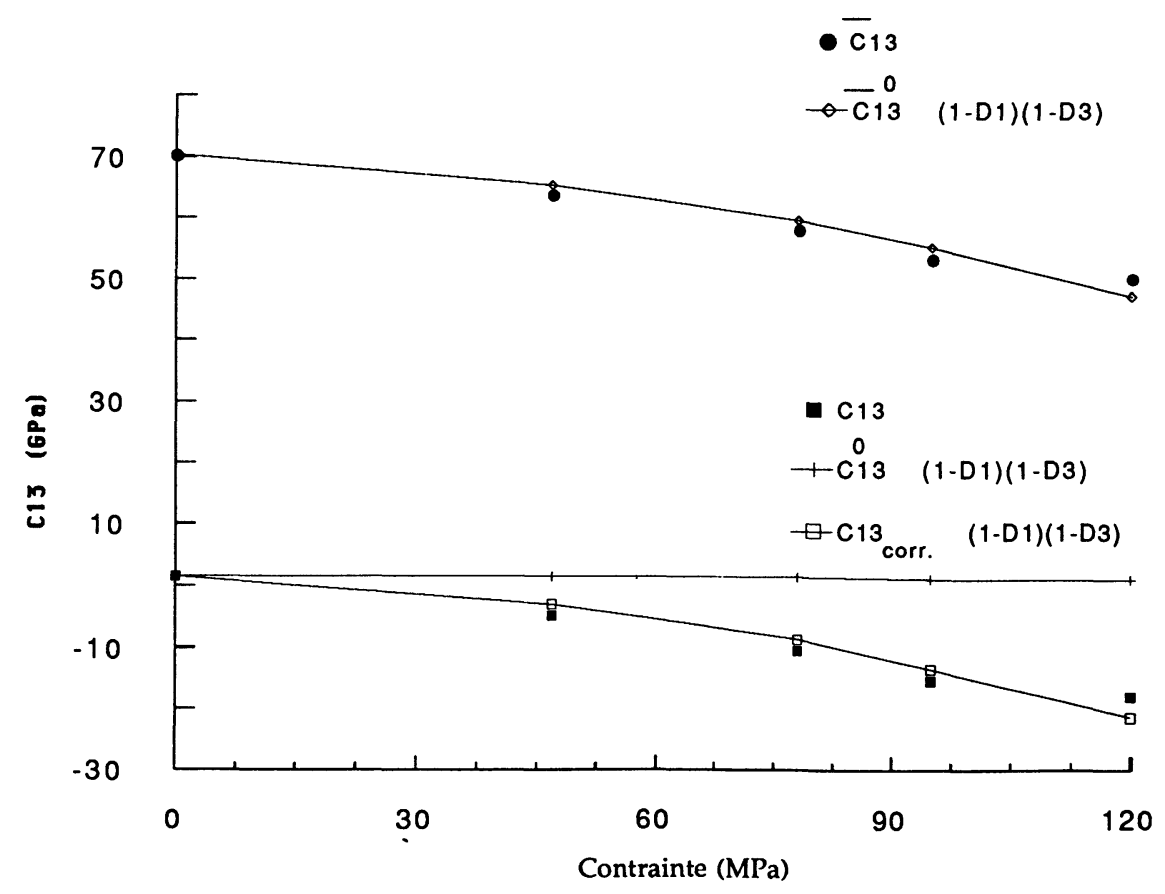

Fig. 11. - Correction du modèle portant sur l'évolution de la raideur $C_{13}$.

[Model correction relative to the $C_{13}$ stiffness evolution.] 
élasticité anisotrope et plasticité induite par l'endommagement [20], sans pour autant que, dans la panoplie de mesures effectuées, une seule donne accès à un contrôle direct de la validité de la modélisation.

Pour les matériaux anisotropes, seuls des essais biaxiaux, en particulier les essais hors axes [21], permettraient de substantiels progrès dans l'identification de l'endommagement tridimensionnel. Finalement, si l'essentiel du comportement monodimensionnel peut être obtenu par des mesures de déformation, aucune information de l'endommagement transversal induit par un essai d'écrouissage (i.e. perpendiculairement à l'axe de la charge) ne paraît actuellement possible [20]. Un moyen de contourner cet écueil est d'imaginer des métrologies autorisant une détermination plus complète des coefficients de la matrice de raideur. C'est précisément le cas des méthodes ultrasonores qui permettent une investigation dans une large plage angulaire d'un plan d'anisotropie donné [8].

La méthode spectro-interférométrique à immersion, utilisée dans ce travail préliminaire, autorise simultanément la détermination complète des quatre constantes d'élasticité d'un plan principal d'un composite. Pour une modélisation simple de l'anisotropie de l'endommagement liée à la structure étudiée, nous avons obtenu, à partir d'une éprouvette, les trois paramètres d'endommagement de ce plan, leur vitesse d'évolution associée et l'assurance de la validité du modèle retenu.

En effet, malgré une transparence acoustique médiocre du matériau due à son caractère dispersif, à la faible épaisseur de l'échantillon et aux vitesses du mode quasi-longitudinal et du mode quasi-transversal proches qui induisent un mélange de ces deux modes et malgré une plage angulaire de mesure réduite liée à la vitesse de propagation importante par rapport à celle de l'eau, l'auscultation ultrasonore de l'éprouvette dans le plan $(1,3)$ a permis d'accéder aux constantes de raideur $C_{11}, C_{33}$, $C_{55}$ et $C_{13}$ à chaque niveau de charge. Ces quatre constantes sont précisément celles qui caractérisent complètement le plan principal $(1,3)$. Par conséquent, disposant de toutes les informations relatives à ce plan, il est, alors, aisé de décrire l'évolution du comportement élastique dans ce plan par une modélisation bidimensionnelle qui, contrairement à un modèle monodimensionnel, introduit réellement un couplage entre les deux directions de ce plan.

Les variations de ces constantes d'élasticité sont importantes, en particulier celle de la raideur dans la direction 1 (axe de chargement). Ces pertes de rigidité sont les mesures macroscopiques (phénoménologiques) de la microfissuration de la matrice (phénomène microstructural), mécanisme fonda- mental du comportement de ce composite fragilefragile. L'endommagement bidimensionnel du plan principal $(1,3)$ est représenté par 3 variables internes : $D_{1}$ (resp. $D_{3}$ ) endommagement de l'axe 1 (resp. 3) et $D_{5}$ l'endommagement dans le plan de cisaillement interlaminaire $(1,3)$. La mesure indirecte de ces paramètres d'endommagement n'utilise que la variation des constantes de raideur diagonales, contrairement aux méthodes de mesure de déformation pour lesquelles les mesures indirectes de l'endommagement reposent sur les variations des coefficients de Poisson (constantes non diagonales de la matrice de raideur). La variation de la constante non diagonale $C_{13}$ n'est plus utile que pour valider la modélisation.

Néanmoins si cette étude, faite par paliers de charge importants (de l'ordre de $40 \mathrm{MPa}$ ) et sur une seule éprouvette déjà précontrainte, a permis l'identification des paramètres d'endommagement $D_{1}$, $D_{3}$ et $D_{5}$ au-delà de $75 \mathrm{MPa}$, elle n'a naturellement pas permis de connaître avec précision le comportement du matériau avant la précharge de $75 \mathrm{MPa}$. Une nouvelle campagne d'essais effectuée par palier de chargement beaucoup plus rapprochés doit être entreprise pour acquérir une meilleure connaissance de ce phénomène de dommage notamment lors des chargements cyclés. En effet, les processus de réouverture de fissures qui accompagnent les remontées en charge peuvent éventuellement conduire à des évolutions brusques des variables internes qui ne peuvent être évaluées que si les paliers de chargement sont suffisamment proches.

Par ailleurs, une investigation dans le plan $(2,3)$ fournirait les constantes $C_{22}, C_{33}, C_{44}$ et $C_{23}$ et donnerait accès à deux nouveaux paramètres d'endommagement $D_{2}$ et $D_{4}$ et à une mesure complémentaire pour valider la modélisation. La détermination du dernier paramètre $D_{6}$, associé à la perte de rigidité en cisaillement translaminaire, demanderait une auscultation du plan $(1,2)$ qui n'est possible que pour des échantillons épais. Ainsi, par ultrasons et pour des composites se présentant habituellement sous forme de plaques minces, on voit qu'il est possible de mesurer 5 des 6 variables internes nécessaires à la description tensorielle de l'endommagement anisotrope (tridimensionnel) ainsi qu'aux vitesses qui leur sont associées.

Enfin, la sensibilité de la méthode donne un espoir certain de pouvoir s'assurer de l'état initial de l'éprouvette (vierge ou non), en terme de vitesse ou même d'amortissement, ce qui, a priori, semble difficile pour les méthodes de mesures mécaniques conventionnelles. Ce contrôle non destructif des éprouvettes permettra de s'affranchir d'un certain nombre de problème de dispersion intrinsèque aux matériaux composites. 


\section{Bibliographie}

[1] Bernhart G., Lamice P., Mace J., Fiabilité des composites céramiques-céramiques, Ind. Céram. 790 (1985) 51-56.

[2] Aveston J., Cooper G. A., Kelly A., Single and multiple fracture, Properties of fiber composites, IPC science and technology press (Guildford, Surrey) 1971 , pp. 15-24.

[3] Birbis J. M., Quenisset J. M., Naslain R., Etude du comportement pseudo-plastique de matériaux composites $2 \mathrm{D}$ carbone-carbone, C. R. $5^{\text {es }}$ journées nationales sur les composites, Paris (Edition Pluralis) 1986, pp. 313-327.

[4] Peres J. P., Anquez L., Jamet F., Analyse micromécanique de la rupture des composites céramiques, Revue Phys. Appl. 23 (1988) 213-228.

[5] INGHels E., LAMON J., Caractérisation de l'endommagement de composites tissés à matrice Sic, Revue Phys. Appl. 23 (1988) 193-200.

[6] BASTe S., GeraRd A., Endommagement anisotrope des composites à matrice céramique. $C$. $R$. Acad. Sci. Paris, Série II, 305 (1987) 1511-1516.

[7] HoSTen B., BARrot A., RouX J., Interférométrie numérique ultrasonore pour la détermination de la matrice de raideur des matériaux composites. Acustica 53 (1983) 212-217.

[8] Roux J., Hosten B., Castagnede B., Deschamps M., Caractérisation mécanique des solides par spectro-interférométrie ultrasonore. Revue Phys. Appl. 20 (1985) 351-358.

[9] Gerard A., Mesure de l'endommagement par méthodes ultrasonores, Rev. F. Mec. 82 (1982) 49-56.

[10] Lemaitre J., Chaboche J. L., Aspect phénoménologique de la rupture par endommagement, $J$. Mec. Appl. 2 (1978) 167-189.

[11] Lemaitre J., Dufailly J., Modélisation et identification de l'endommagement plastique des métaux, $3^{\mathrm{e}}$ Congrès français de mécanique, Grenoble (1977).
[12] Cordebois J. P., SidorofF F., Endommagement anisotrope en élasticité et plasticité, J. Mec. Theor. Appl., numéro spécial (1982) 45-60.

[13] Chaboche J. L., Le concept de contrainte effective appliqué à l'élasticité et à la viscoplasticité en présence d'un endommagement anisotrope, Comportement mécanique des solides anisotropes, Ed. J. P. Boehler (EuroMECH, Villard de Lens) 1979.

[14] CoRdebois J. P., SidorofF F., Damage Induced Elastic Anisotropy, Comportement mécanique des solides anisotropes, Ed. J. P. Boehler (EuroMECH, Villard de 'Lens) 1979.

[15] Deschamps M., Etude en ondes planes et faisceaux bornés de la réfraction à une interface liquidesolide, application à la caractérisation viscoélastique des composites, Thèse de l'Université de Bordeaux I (1985).

[16] Auld B. A., Acoustic fields and waves in solids (Wiley-Interscience, New York) Vol. 1, 1973.

[17] Hosten B., Castagnede B., Optimisation des constantes élastiques à partir des mesures de vitesses d'une onde ultrasonore, C. R. Acad. Sci. Paris 296, Série II (1983) 297-300.

[18] Castagnede B., Mesure des constantes élastiques de solides anisotropes par une méthode ultrasonore, Thèse $3^{e}$ cycle, Bordeaux I (1984).

[19] BASTE S., Damage evaluation of ceramic-ceramic composite in tension by means of deformation measurements, à paraître.

[20] Baste S., Charcosset C., Sidoroff F., Modélisation élasto-plastique endommageable d'un composite 3D, C. R. $6^{\text {es }}$ journées nationales sur les composites, Paris, 11-13 octobre 1988.

[21] Ladeveze P., Sur la mécanique de l'endommagement des composites, C. R. $5^{\text {es }}$ journées nationales sur les composites, Paris (Edition Pluralis) 1986, pp. 685-697. 